\title{
Diseño y evaluación de proyectos educativos en el nivel superior (Pontificia Universidad Católica del Ecuador, Sede Santo Domingo)
}

\author{
Design and evaluation of educational projects at higher education
}

\author{
Lenin Mendieta ${ }^{1}$; Darwin García ${ }^{2}$; \\ Sandra Chung ${ }^{3}$; Rijkaard Mendieta ${ }^{4}$
}

http://dx.doi.org/10.21503/CienciayDesarrollo.2014.v17i2.05

\section{RESUMEN}

El presente trabajo de investigación tiene su objeto de estudio el diseño curricular y el campo de la evaluación de planes y proyectos educativos, el mismo pretendió establecer una ruta de aprendizaje de la competencia de la comunicación oral en público a los estudiantes de la Escuela de Ciencias de la Educación de la Pontificia Universidad Católica del Ecuador - Sede Santo Domingo. Para ello se diseñó un proyecto educativo denominado "Conversatorio Pedagogía 3000", el cual tuvo su sustento en la entrevista a 120 estudiantes de la escuela y posterior encuesta in situ a 42 estudiantes sobre el miedo escénico de hablar en público y la pertinencia de realizar este tipo de eventos pedagógicos. Las conclusiones a las que se llegó: existen limitaciones a la hora de hablar en público de parte de los estudiantes con un porcentaje de hasta el $85 \%$, sin embargo un $43 \%$ consideró no ser necesarios este tipo de eventos, contraponiéndose a la encuesta realizada luego del conversatorio, la cual indica que un $80,95 \%$ quedo muy satisfecho con el evento.

Palabras clave: Diseño curricular, conversatorio pedagogía.

\section{ABSTRACT}

This research has its purpose of curriculum design study, besides educative plans and projects in evaluation fields; it attempts to stablish a learning method about competence in oral communication in front of a public auditorium, guided to Pontificia Universidad Catolica del Ecuador, Sede Santo Domingo, College of Education students. To perform this research, an educative project called "Conversatorio Pedagogía 3000" was designed; the study had support in the interview to 120 college students and subsequent survey was applied in situ to 42 college students about scenic fear of public speaking and relevance of this type of educational events. The conclusions to which were reached is that there are bounds in public speaking from students with a percentage up to $85 \%$, but up to $43 \%$ considered these events are not necessary being opposed to the survey conducted after the discussion group which indicates that up to $80.95 \%$ was very satisfied with the event.

Keywords: Curriculum design, conversatorio pedagogía.

\footnotetext{
1 Docente-Investigador a tiempo completo en la Pontifica Universidad Católica del Ecuador, Escuela de Ciencias de la Educación.

2 Docente-Investigador a tiempo completo en la Pontifica Universidad Católica del Ecuador, Escuela de Ciencias de la Educación.

Docente-Investigador a tiempo completo en la Pontifica Universidad Católica del Ecuador, Escuela de Ciencias de la Educación.

4 Estudiante-co-investigador en la Universidad Técnica Particular de Loja (UTPL).
} 


\section{INTRODUCCIÓN}

El objeto en el que se enmarca el presente artículo es el del diseño curricular y el campo de la evaluación de planes y proyectos educativos. Conforme cita Frida Díaz Barriga (1997:7) “Uno de los factores determinantes en el desarrollo social de un país es la educación: por tanto, ésta debe contribuir al logro de los objetivos socialmente válidos, para lo cual se apoya en la planeación” y, de acuerdo a Villarreal (1980:7). Planear es un acto de inteligencia cuyo propósito es racionalizar la selección de alternativas para el futuro. Implica un ejercicio libre de la razón para definir con claridad los fines a los que se orienta la acción, y desentrañar los mejores medios para alcanzarlos.

Si como juicio racional y coherente, se aplican estos criterios bien enunciados por los actores intelectuales de los mismos y, sumamos el citado por Kenneth Delgado (2008, pág. 8) sobre evaluación en la educación superior “...al contrario, reducen la problemática de la evaluación educacional a un asunto de rendimiento, no la comprenden de una manera integral", haciendo referencia a este enunciado y extrapolando el mismo a la evaluación de planes $y / o$ proyectos educativos, se puede inferir que ha existido una errónea conceptualización sobre la forma de evaluar dichos insumos académicos.

La comunicación oral es un instrumento para comunicar sobre procesos u objetos externos a él, en el plano educativo es la herramienta más importante por cuanto con ella se llega o no a los alumnos, esta competencia es más amplia que el habla, la misma requiere de elementos paralingüísticos para completar su significación final, que no solo implica un conocimiento adecuado del idioma, abarca también varios elementos no verbales. Mehrabian, citado por Orozco E (1996, pág.3), identificó que el impacto total de un mensaje es aproximadamente un 7\% verbal, un $38 \%$ vocal (tono de voz, ritmo, etc.) y un $55 \%$ no verbal.

De igual manera, Givens (2000:4) cita "Cuando hablamos (o escuchamos), nuestra atención se centra en las palabras más que en el lenguaje corporal. Aunque nuestro juicio incluye ambas cosas. Una audiencia está procesando simultáneamente el aspecto verbal y el no verbal. Los movimientos del cuerpo no son generalmente positivos o negativos en sí mismos, más bien, la situación y el mensaje determinarán su evaluación."

En el diseño y evaluación de proyectos educativos en el nivel superior, en la Pontificia Universidad Católica del Ecuador, sede Santo Domingo, en la escuela de ciencias de la educación, se realizó en primer lugar una evaluación sobre la competencia de la comunicación oral para el desenvolvimiento eficaz de la actividad del futuro profesor, se llegó a la conclusión que era necesario planificar proyectos que induzcan al futuro docente a "perder el miedo escénico"* , de hablar en público.

Luego de la evaluación y su diagnóstico se pudo evidenciar una limitación de esta competencia y se diseñó el proyecto "Conversatorio Pedagogía 3000", que fue realizado en aras de construir un espacio donde los estudiantes puedan explayar sus inquietudes, necesidades y temas pedagógicos que ellos considerasen pertinentes.

El objetivo general del proyecto fue el de lograr la adquisición dela competencia dela comunicación oral de los estudiantes. Los objetivos específicos fueron: Crear y fortalecer espacios de diálogo y debate en ECE; establecer el grado de satisfacción de los asistentes al conversatorio, para lo cual se generó una encuesta realizada in situ.

* Gabriel García Márquez la utilizaba para describir el pánico que sentía cuando se veía obligado a hablar en público 


\section{MATERIAL Y MÉTODO}

Participaron 42 estudiantes de la carrera de ciencias de la educación quienes fueron sujetos de una encuesta sobre el grado de satisfacción de los mismos, aquí se recogió todos los parámetros e indicadores para la evaluación del proyecto.

Para poder establecer el diagnóstico se realizó una entrevista a 120 estudiantes de la misma carrera con la pregunta si tenían miedo de participar en público en coloquios y/o conversatorios y si creían que eran necesarios espacios donde se puedan solventar algunas limitaciones de hablar en público

Una vez diseñado el proyecto y para el desarrollo del conversatorio se siguió la siguiente metodología: Preparación, Selección de panelistas, Presentación de ponencias, Aclaraciones y preguntas, Reflexión sobre la temática, Síntesis

La encuesta tuvo los siguientes datos: De información general (Organización y planificación del evento, Difusión del evento, Facilidad para trámites administrativos: inscripción, Información o comunicaciones del evento, Agilidad en el proceso administrativo del evento, Atención del personal organizador del evento); Logística (Fácil acceso al lugar del evento, Calidad y comodidad de las instalaciones del lugar del evento, Interés de las actividades paralelas al evento (entrega de material, registro de asistencia, entrega de premios, etc.), La funcionalidad de espacio disponible, El ambiente de trabajo en que se desarrollaron las actividades programadas, El audio y sonido durante la intervención); Programación (Pertinencia de las conferencias organizadas (inauguración, clausura y sesiones plenarias), Pertinencia de las áreas temáticas establecidas, Grado de interés de las ponencias expuestas, Innovación en los temas presentados, Calidad en la moderación (control de tiempos, turnos de palabra, etc.), Cumplimiento de la programación establecida); Valoración general (Grado de satisfacción general del evento); una pregunta suelta de ¿Considera usted que es necesario realizar este tipo de eventos para la formación profesional de los futuros docentes? y Comentarios y sugerencias.

\section{RESULTADOS}

Para llevar a cabo un análisis de forma más clara, se creó un archivo en Microsoft Excel, donde se realizó el vaciado de los datos obtenidos en la entrevista (120 en total) y en las encuestas ( 42 en total), para posteriormente ser analizados por medio de las tablas y gráficos que se detallan a continuación.

\section{Encuesta de diagnóstico sobre el proyecto}

Tabla 1

$\begin{array}{lc}\text { PARÁMETROS } & \begin{array}{c}\text { ¿Tiene miedo de hablar en } \\ \text { público? }\end{array} \\ \text { SI } & \mathbf{1 0 2} \\ \text { NO } & \mathbf{1 0} \\ \text { UN POCO } & \mathbf{8}\end{array}$

Fuente: Estudiantes de la carrera de Ciencias de la Educación Elaborado por: Lenin Mendieta Toledo

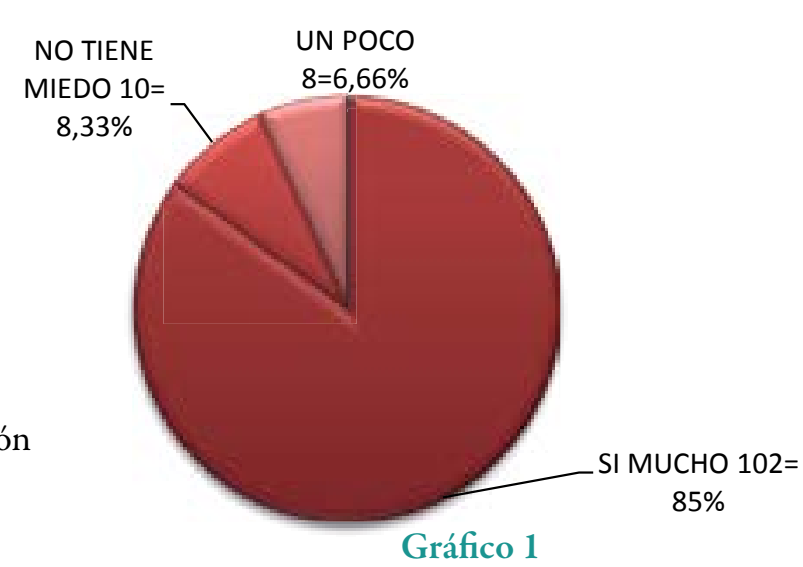


Tabla 2

$\begin{array}{lc}\text { PARÁMETROS } & \begin{array}{c}\text { ¿Son necesarios eventos } \\ \text { para perder el miedo de } \\ \text { hablar en público? }\end{array} \\ \text { SI } & 68 \\ \text { NO } & 52\end{array}$

Fuente: Estudiantes de la carrera de Ciencias de la Educación Elaborado por: Lenin Mendieta Toledo

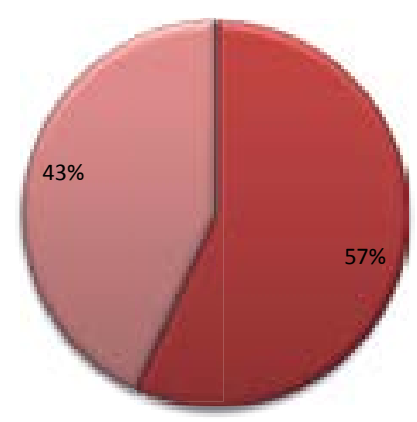

Gráfico 2

\section{Información general del evento}

Tabla 3. Organización y planificación del evento

\begin{tabular}{lcc}
\multicolumn{1}{c}{ PARÁMETROS } & N & $\%$ \\
\hline Excelente & 22 & 52 \\
\hline Muy Bueno & 18 & 43 \\
Bueno & 2 & 5 \\
\hline Regular & 0 & 0 \\
Malo & 0 & 0 \\
\hline Total & $\mathbf{4 2}$ & $\mathbf{1 0 0 \%}$
\end{tabular}

Fuente: Estudiantes de la carrera de Ciencias de la Educación

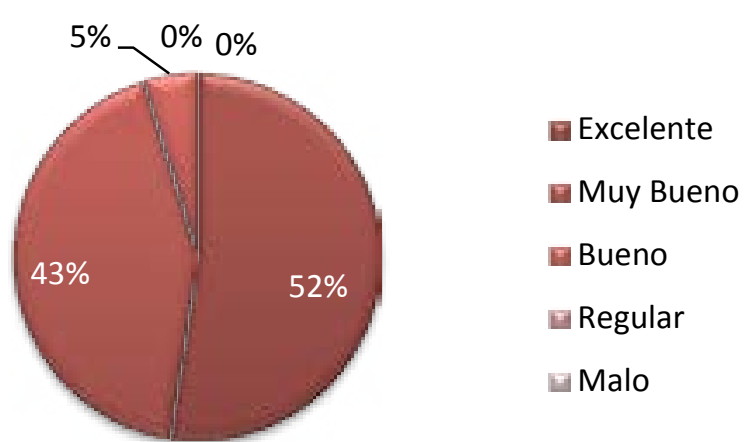

Elaborado por: Lenin Mendieta Toledo

Gráfico 3

En cuanto a la organización y planificación del evento, un 52\% dio una valoración de excelente, el $43 \%$ muy bueno y, el $5 \%$ bueno.

Tabla 4. Difusión del evento

\begin{tabular}{lll}
\multicolumn{1}{c}{ PARÁMETROS } & \multicolumn{1}{c}{$\mathbf{N}^{\circ}$} & \multicolumn{1}{c}{$\%$} \\
\hline Excelente & 19 & 45 \\
Muy Bueno & 15 & 36 \\
Bueno & 6 & 14 \\
Regular & 2 & 5 \\
Malo & 0 & 0 \\
Total & 42 & $100 \%$
\end{tabular}

Fuente: Estudiantes de la carrera de Ciencias de la Educación

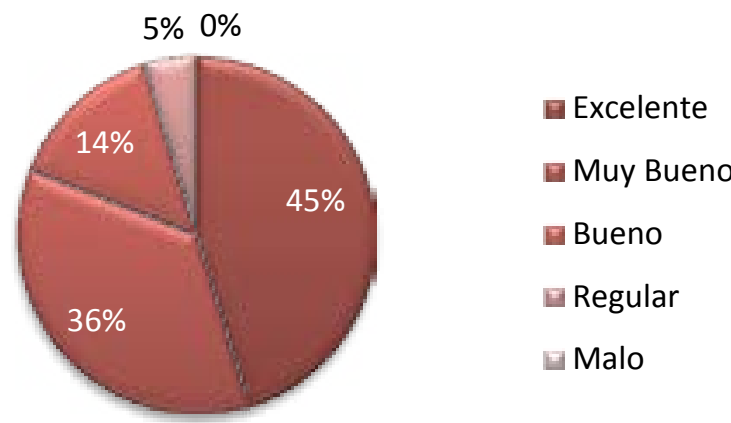

Gráfico 4

Elaborado por: Lenin Mendieta Toledo

En cuanto a la difusión del evento, un 45\% dio una valoración de excelente, el 36\% muy bueno, el 14\% bueno y, el $5 \%$ regular. 
Tabla 5. Facilidad para trámites administrativos: inscripción

\begin{tabular}{lll}
\hline PARÁMETROS & $\mathbf{N}^{\circ}$ & $\%$ \\
\hline Excelente & 24 & 57 \\
Muy Bueno & 10 & 24 \\
Bueno & 6 & 14 \\
\hline Regular & 2 & 5 \\
Malo & 0 & 0 \\
\hline Total & $\mathbf{4 2}$ & $\mathbf{1 0 0 \%}$
\end{tabular}

Fuente: Estudiantes de la carrera de Ciencias de la Educación Elaborado por: Lenin Mendieta Toledo

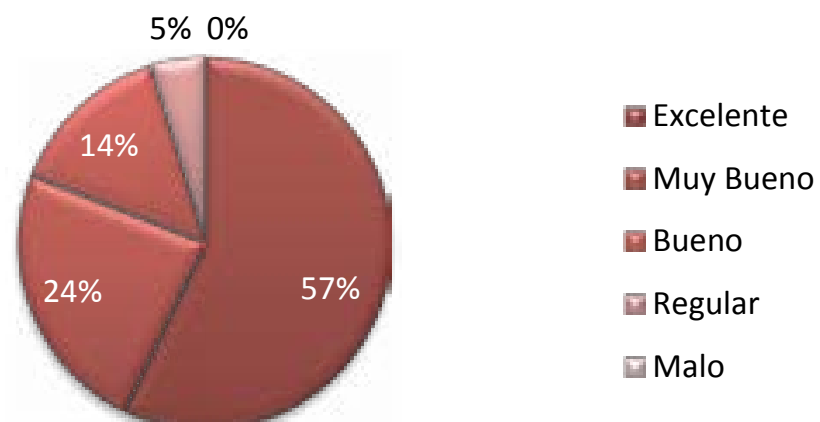

En cuanto a la facilidad para trámites administrativos: inscripción, un $57 \%$ dio una valoración de excelente, el $24 \%$ muy bueno y, el $14 \%$ bueno y, el $5 \%$ regular.

Tabla 6. Información o comunicaciones del evento.

\begin{tabular}{|lll}
\multicolumn{1}{c}{ PARÁMETROS } & \multicolumn{1}{c}{ No $^{\circ}$} \\
\hline Excelente & 23 & 55 \\
Muy Bueno & 8 & 19 \\
Bueno & 11 & 26 \\
Regular & 0 & 0 \\
Malo & 0 & 0 \\
\hline Total & $\mathbf{4 2}$ & $\mathbf{1 0 0 \%}$
\end{tabular}

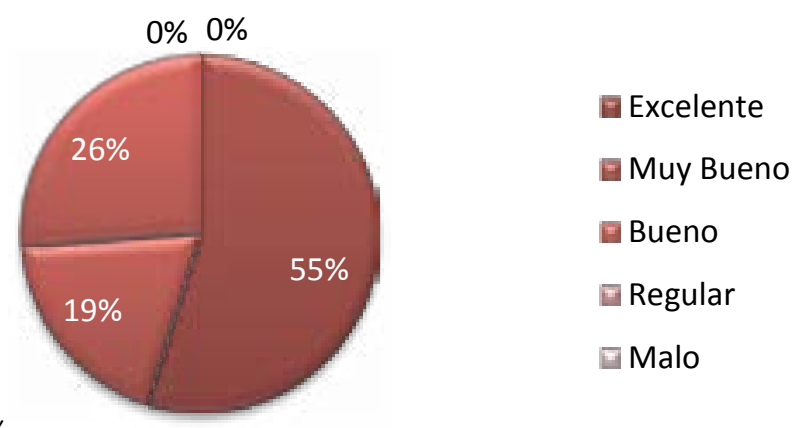

Fuente: Estudiantes de la carrera de Ciencias de la Educación Elaborado por: Lenin Mendieta Toledo

Gráfico 3

En cuanto a la Información o comunicaciones del evento, un 55\% dio una valoración de excelente, el $19 \%$ muy bueno y, el $26 \%$ bueno.

Tabla 7. Agilidad en el proceso administrativo del evento.

\begin{tabular}{lll}
\multicolumn{1}{c}{ PARÁMETROS } & \multicolumn{1}{c}{$\mathbf{N}^{\circ}$} & \multicolumn{1}{c}{$\%$} \\
\hline Excelente & 21 & 50 \\
Muy Bueno & 10 & 24 \\
Bueno & 9 & 21 \\
Regular & 2 & 5 \\
Malo & 0 & 0 \\
\hline Total & $\mathbf{4 2}$ & $\mathbf{1 0 0 \%}$
\end{tabular}

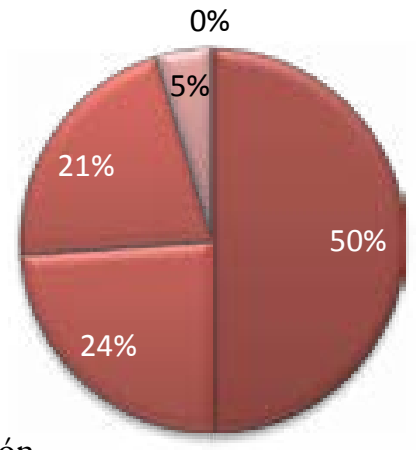

Excelente

Muy Bueno

Bueno

Regular

$\square$ Malo

Fuente: Estudiantes de la carrera de Ciencias de la Educación

Elaborado por: Lenin Mendieta Toledo.

Gráfico 4

En cuanto en la agilidad en el proceso administrativo del evento, un 50\% dio una valoración de excelente, el $24 \%$ muy bueno, el $21 \%$ bueno y, el $5 \%$ bueno. 
Tabla 8. Atención del personal organizador del evento.

\begin{tabular}{lcc}
\multicolumn{1}{c}{ PARÁMETROS } & No $^{\circ}$ & $\%$ \\
\hline Excelente & 23 & 55 \\
Muy Bueno & 12 & 29 \\
Bueno & 6 & 14 \\
Regular & 1 & 2 \\
Malo & 0 & 0 \\
Total & $\mathbf{4 2}$ & $\mathbf{1 0 0 \%}$
\end{tabular}

Fuente: Estudiantes de la carrera de Ciencias de la Educación

Elaborado por: Lenin Mendieta Toledo

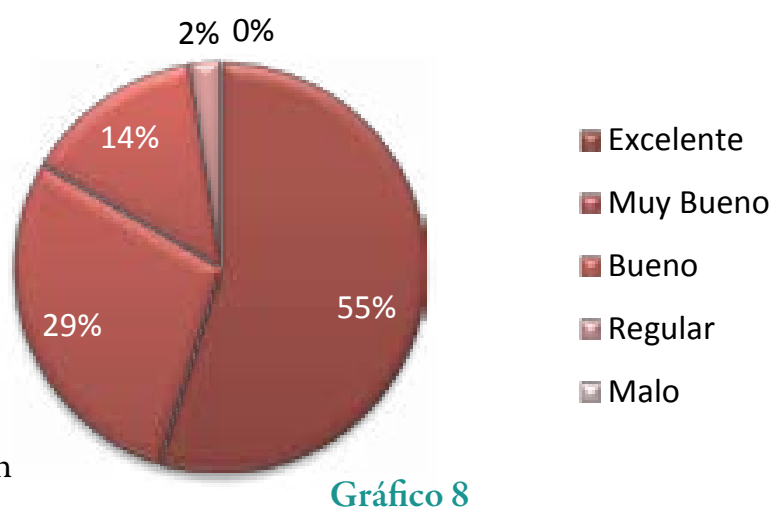

En cuanto a la atención del personal organizador del evento, un 55\% dio una valoración de excelente, el $29 \%$ muy bueno, el $14 \%$ bueno y, $2 \%$ regular.

Tabla 9. Fácil acceso al lugar del evento.

\begin{tabular}{lcc}
\multicolumn{1}{c}{ PARÁMETROS } & $N^{\circ}$ & $\%$ \\
\hline Excelente & 36 & 86 \\
Muy Bueno & 5 & 12 \\
Bueno & 1 & 2 \\
Regular & 0 & 0 \\
Malo & 0 & 0 \\
Total & $\mathbf{4 2}$ & $\mathbf{1 0 0 \%}$
\end{tabular}

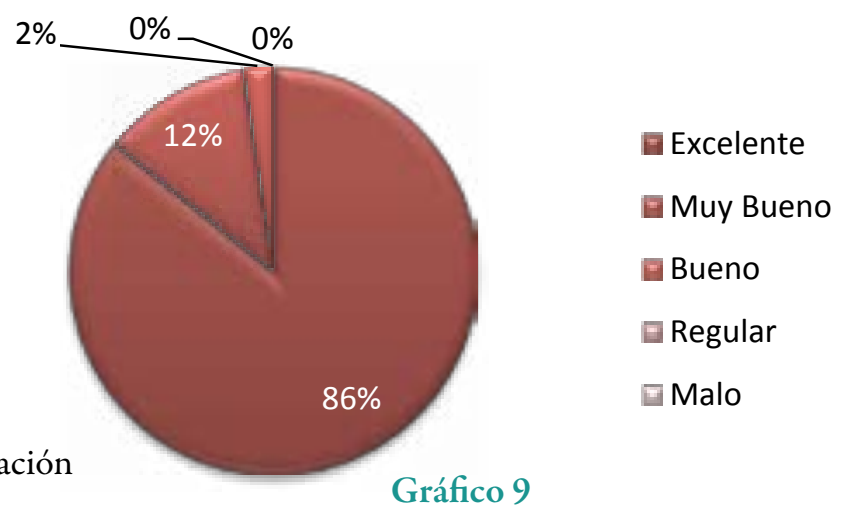

Elaborado por: Lenin Mendieta Toledo

En cuanto al fácil acceso al lugar del evento, un $86 \%$ dio una valoración de excelente, el $12 \%$ muy bueno y, el $2 \%$ bueno.

Tabla 10. Calidad y comodidad de las instalaciones del lugar del evento.

\begin{tabular}{lcc}
\multicolumn{1}{c}{ PARÁMETROS } & No $^{\circ}$ & $\%$ \\
\hline Excelente & 38 & 90 \\
Muy Bueno & 4 & 10 \\
Bueno & 0 & 0 \\
Regular & 0 & 0 \\
Malo & 0 & 0 \\
\hline Total & $\mathbf{4 2}$ & $\mathbf{1 0 0 \%}$
\end{tabular}

Fuente: Estudiantes de la carrera de Ciencias de la Educación Elaborado por: Lenin Mendieta Toledo

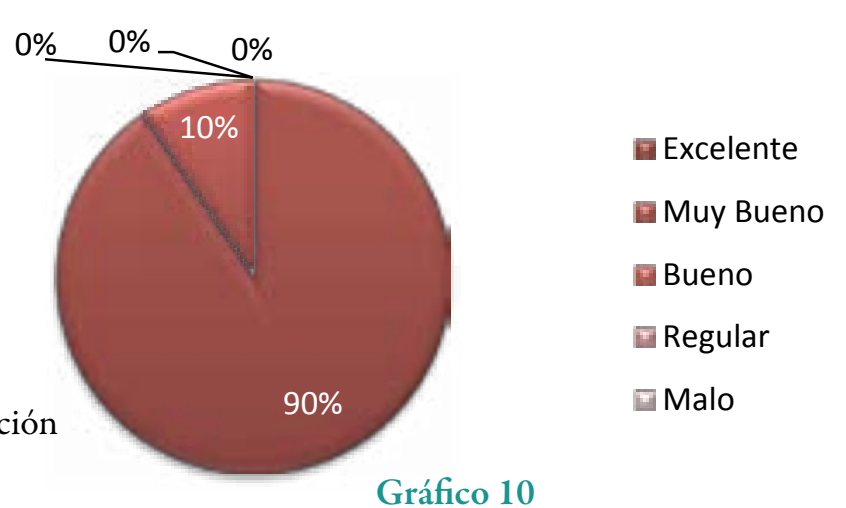

En cuanto a la calidad y comodidad de las instalaciones del lugar del evento, un 90\% dio una valoración de excelente, el 10\% muy bueno. 
Tabla 11. Interés de las actividades paralelas al evento (entrega de material, registro de asistencia, entrega de premios, etc.)

\begin{tabular}{lcc}
\multicolumn{1}{c}{ PARÁMETROS } & No & $\%$ \\
\hline Excelente & 24 & 57 \\
Muy Bueno & 14 & 33 \\
Bueno & 3 & 7 \\
Regular & 1 & 2 \\
Malo & 0 & 0 \\
Total & $\mathbf{4 2}$ & $\mathbf{1 0 0 \%}$
\end{tabular}

Fuente: Estudiantes de la carrera de Ciencias de la Educación

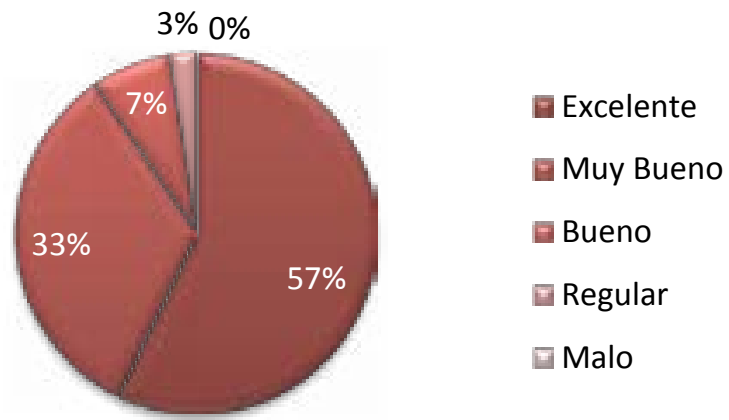

Elaborado por: Lenin Mendieta Toledo

Gráfico 11

En cuanto al interés de las actividades paralelas al evento (entrega de material, registro de asistencia, entrega de premios, etc.), un 57\% dio una valoración de excelente, el 33\% muy bueno, el $7 \%$ bueno y, el $3 \%$ regular.

Tabla 12. La funcionalidad de espacio disponible.

\begin{tabular}{lcc}
\multicolumn{1}{c}{ PARÁMETROS } & No $^{\circ}$ & $\%$ \\
\hline Excelente & 34 & 81 \\
\hline Muy Bueno & 4 & 10 \\
Bueno & 4 & 10 \\
Regular & 0 & 0 \\
Malo & 0 & 0 \\
\hline Total & $\mathbf{4 2}$ & $\mathbf{1 0 0 \%}$
\end{tabular}

Fuente: Estudiantes de la carrera de Ciencias de la Educación Elaborado por: Lenin Mendieta Toledo

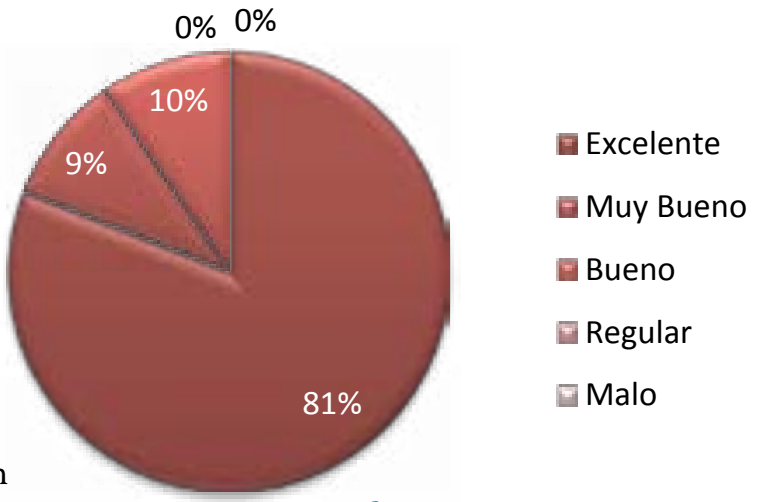

Gráfico 12

En cuanto a la funcionalidad de espacio disponible, un $81 \%$ dio una valoración de excelente, el 9\% muy bueno y, el 10\% bueno.

Tabla 13. El ambiente de trabajo en que se desarrollaron las actividades programadas.

\begin{tabular}{lcc}
\multicolumn{1}{c}{ PARÁMETROS } & N $^{\circ}$ & $\%$ \\
\hline Excelente & 26 & 62 \\
\hline Muy Bueno & 9 & 21 \\
Bueno & 7 & 17 \\
Regular & 0 & 0 \\
Malo & 0 & 0 \\
\hline Total & $\mathbf{4 2}$ & $\mathbf{1 0 0 \%}$
\end{tabular}

Fuente: Estudiantes de la carrera de Ciencias de la Educación

Elaborado por: Lenin Mendieta Toledo



En cuanto al ambiente de trabajo en que se desarrollaron las actividades programadas, un $62 \%$ dio una valoración de excelente, el 21\% muy bueno y, el 17\% bueno. 
Tabla 14. El audio y sonido durante la audición.

\begin{tabular}{lcc}
\multicolumn{1}{c}{ PARÁMETROS } & No & $\%$ \\
\hline Excelente & 30 & 71 \\
Muy Bueno & 9 & 21 \\
Bueno & 3 & 7 \\
Regular & 0 & 0 \\
Malo & 0 & 0 \\
Total & $\mathbf{4 2}$ & $\mathbf{1 0 0 \%}$
\end{tabular}

Fuente: Estudiantes de la carrera de Ciencias de la Educación

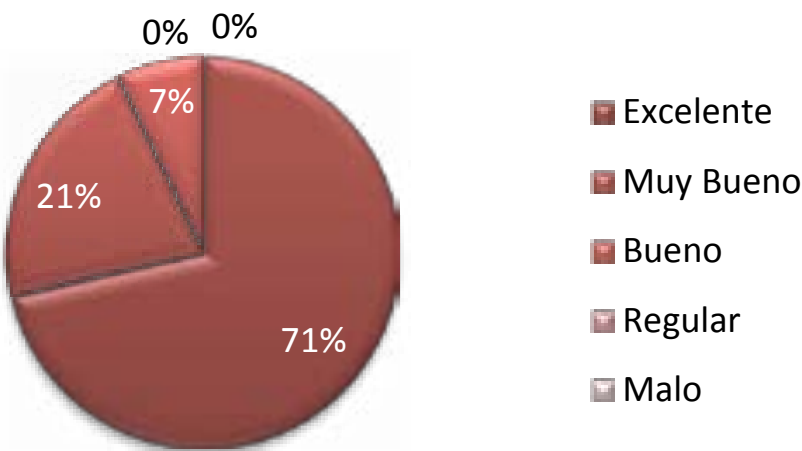

Elaborado por: Lenin Mendieta Toledo

Gráfico 14

En cuanto al audio y sonido durante la audición, un $71 \%$ dio una valoración de excelente, el $21 \%$ muy bueno y, el $7 \%$ bueno.

Tabla 15. Pertinencia de las conferencias organizadas (inauguración, clausura y sesiones plenarias)

\begin{tabular}{lcc}
\multicolumn{1}{c}{ PARÁMETROS } & $\mathbf{N}^{\circ}$ & $\%$ \\
\hline Excelente & 25 & 59 \\
Muy Bueno & 15 & 36 \\
Bueno & 2 & 5 \\
Regular & 0 & 0 \\
Malo & 0 & 0 \\
Total & $\mathbf{4 2}$ & $\mathbf{1 0 0 \%}$
\end{tabular}

Fuente: Estudiantes de la carrera de Ciencias de la Educación Elaborado por: Lenin Mendieta Toledo

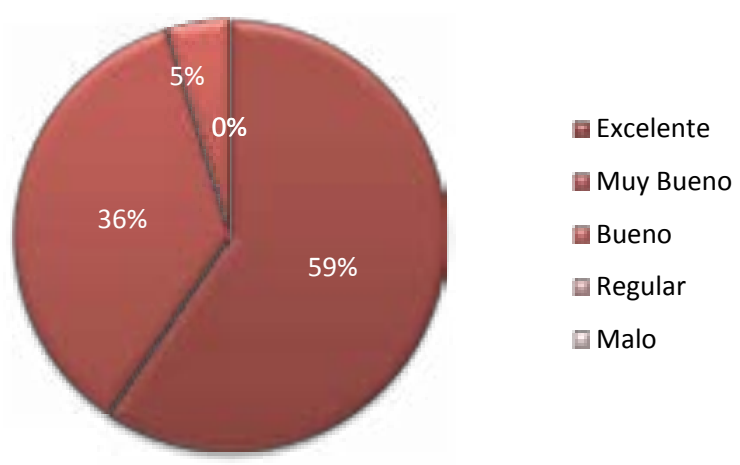

Gráfico 15

En cuanto a la pertinencia de las conferencias organizadas (inauguración, clausura y sesiones plenarias), un 59\% dio una valoración de excelente, el 36\% muy bueno y, el $5 \%$ bueno.

Tabla 16. Pertinencia de las áreas temáticas establecidas.

\begin{tabular}{lcc}
\multicolumn{1}{c}{ PARÁMETROS } & $\mathbf{N}^{\circ}$ & $\%$ \\
\hline Excelente & 22 & 52 \\
Muy Bueno & 19 & 45 \\
Bueno & 1 & 3 \\
Regular & 0 & 0 \\
Malo & 0 & 0 \\
Total & $\mathbf{4 2}$ & $\mathbf{1 0 0 \%}$
\end{tabular}

Fuente: Estudiantes de la carrera de Ciencias de la Educación Elaborado por: Lenin Mendieta Toledo

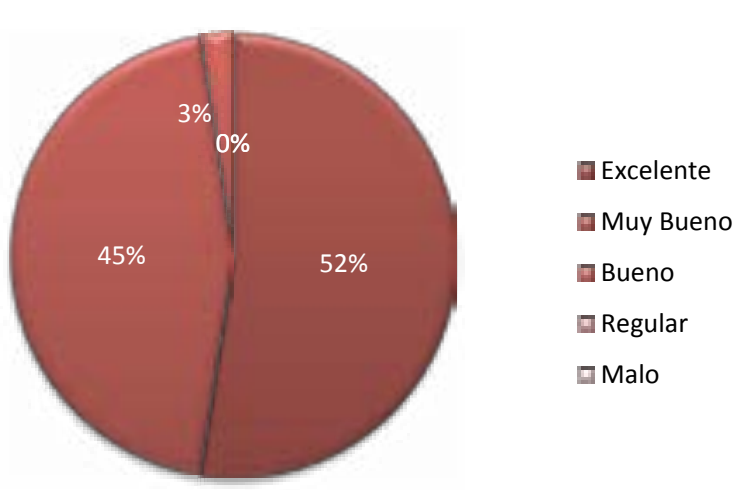

Gráfico 16

En cuanto a la pertinencia de las áreas temáticas establecidas, un 52\% dio una valoración de excelente, el $45 \%$ muy bueno y, el $3 \%$ bueno. 
Tabla 17. Grado de interés de las ponencias expuestas.

\begin{tabular}{lcc}
\multicolumn{1}{c}{ PARÁMETROS } & $\mathbf{N}^{\circ}$ & $\%$ \\
\hline Excelente & 23 & 55 \\
\hline Muy Bueno & 14 & 33 \\
Bueno & 5 & 12 \\
Regular & 0 & 0 \\
Malo & 0 & 0 \\
\hline Total & $\mathbf{4 2}$ & $\mathbf{1 0 0 \%}$
\end{tabular}

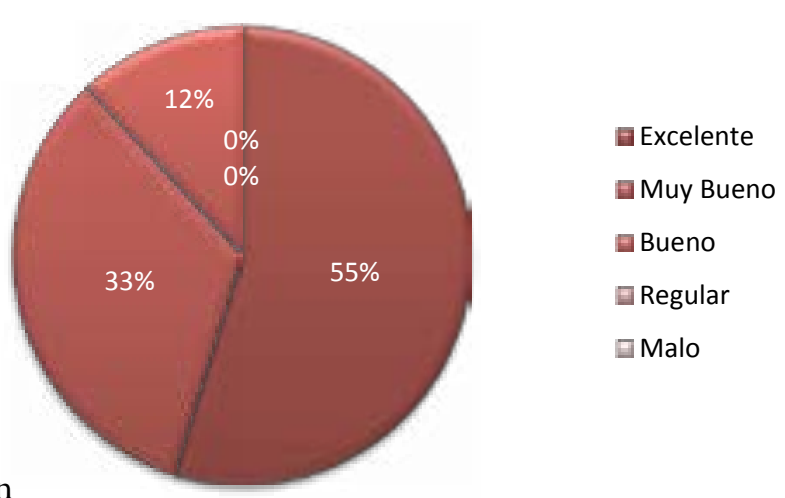

Fuente: Estudiantes de la carrera de Ciencias de la Educación

Elaborado por: Lenin Mendieta Toledo

Gráfico 17

En cuanto al grado de interés de las ponencias expuestas, un 55\% dio una valoración de excelente, el $33 \%$ muy bueno y, el $12 \%$ bueno.

Tabla 18. Innovación de los temas presentados

\begin{tabular}{lcc}
\multicolumn{1}{c}{ PARÁMETROS } & $\mathbf{N}^{\circ}$ & $\%$ \\
\hline Excelente & 24 & 57 \\
Muy Bueno & 14 & 33 \\
Bueno & 4 & 10 \\
Regular & 0 & 0 \\
Malo & 0 & 0 \\
Total & $\mathbf{4 2}$ & $\mathbf{1 0 0 \%}$
\end{tabular}

Fuente: Estudiantes de la carrera de Ciencias de la Educación Elaborado por: Lenin Mendieta Toledo

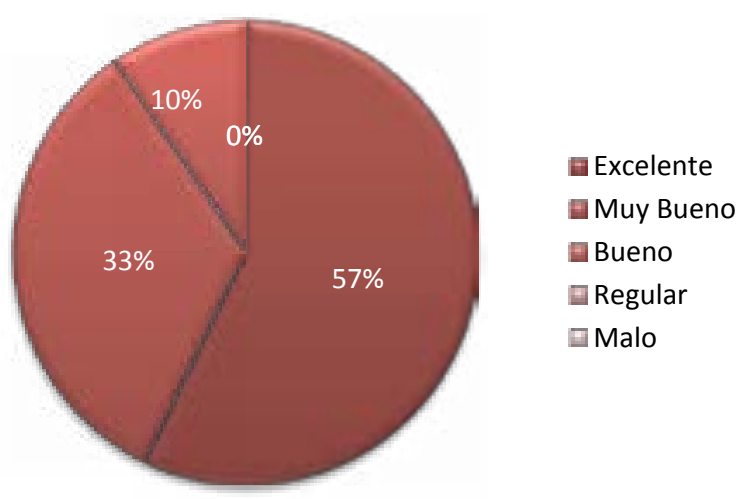

Gráfico 18

En cuanto a la innovación de los temas presentados, un 57\% dio una valoración de excelente, el 33\% muy bueno y, el 10\% bueno.

Tabla 19. Calidad de la moderación (control de tiempos, turnos de palabras, etc.)

\begin{tabular}{lcc}
\multicolumn{1}{c}{ PARÁMETROS } & $\mathbf{N}^{\circ}$ & $\%$ \\
\hline Excelente & 23 & 55 \\
Muy Bueno & 9 & 21 \\
Bueno & 7 & 17 \\
Regular & 3 & 7 \\
Malo & 0 & 0 \\
\hline Total & $\mathbf{4 2}$ & $\mathbf{1 0 0 \%}$
\end{tabular}

Fuente: Estudiantes de la carrera de Ciencias de la Educación Elaborado por: Lenin Mendieta Toledo

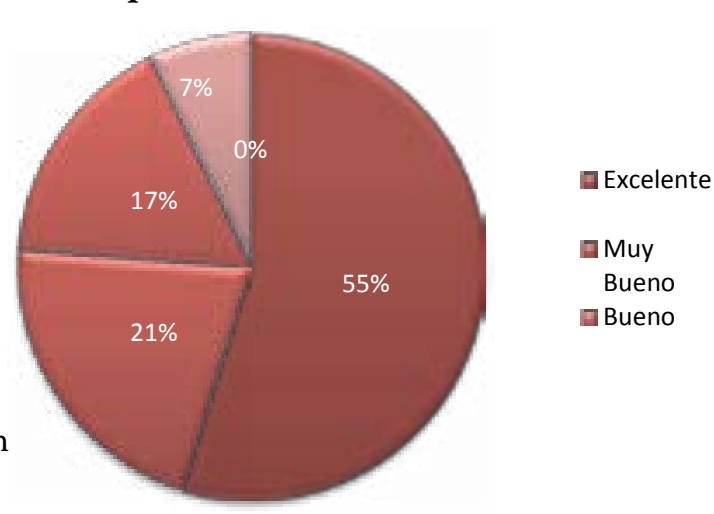

Gráfico 19

En cuanto a la calidad de la moderación (control de tiempos, turnos de palabras, etc.), un 55\% dio una valoración de excelente, el $21 \%$ muy bueno, el $17 \%$ bueno y, el $7 \%$ regular. 
Tabla 20. Cumplimiento de la programación establecida

\begin{tabular}{lcc}
\multicolumn{1}{c}{ PARÁMETROS } & $\mathbf{N}^{\circ}$ & $\%$ \\
\hline Excelente & 28 & 67 \\
Muy Bueno & 9 & 21 \\
Bueno & 5 & 12 \\
Regular & 0 & 0 \\
Malo & 0 & 0 \\
Total & $\mathbf{4 2}$ & $\mathbf{1 0 0 \%}$
\end{tabular}

Fuente: Estudiantes de la carrera de Ciencias de la Educación

Elaborado por: Lenin Mendieta Toledo

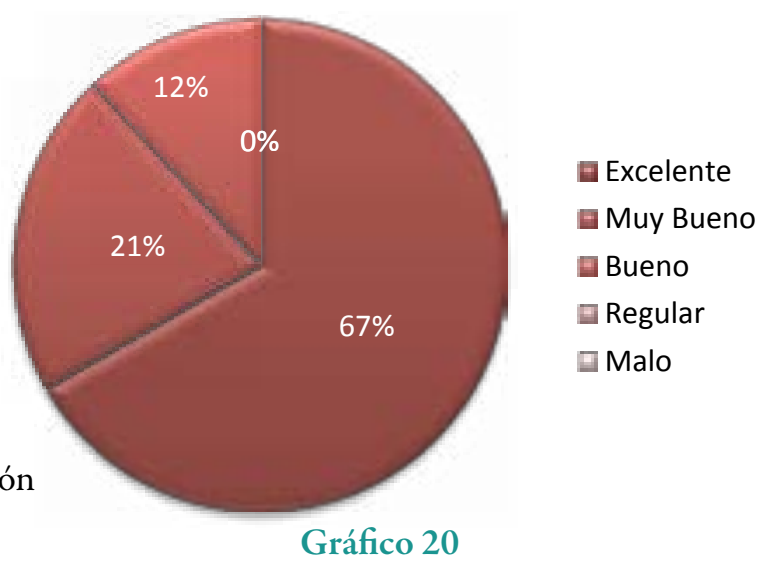

En cuanto al cumplimiento de la programación establecida, un 67\% dio una valoración de excelente, el $21 \%$ muy bueno y, el $12 \%$ bueno.

Tabla 21. Grado de satisfacción general del evento



En cuanto al grado de satisfacción general del evento, un 80,95\% dio una valoración de excelente, el $19,05 \%$ muy bueno.

\section{CONCLUSIONES}

Las conclusiones a las que se ha llegado son que, existen limitaciones en la competencia de comunicación oral en público de parte de los estudiantes de la Escuela de Ciencias de la Educación de la Pontifica Universidad Católica del Ecuador, sede Santo Domingo, que el diseño de proyectos educativos son las herramientas válidas para un mejor desarrollo del proceso educativo, que los estudiantes de la carrera de Ciencias de la Educación tienen un elevado porcentaje de miedo escénico llegando esta cifra hasta un porcentaje altamente significativo del $85 \%$ y que existe una diferencia altamente significativa entre la entrevista con un $43 \%$ que indica que no son necesarios este tipo de proyectos (antes del evento) y la encuesta después de realizado que indica que hasta en un $80,95 \%$ tienen un grado de satisfacción con el proyecto ejecutado.

\section{RECOMENDACIONES}

Construir en base a situaciones reales diseños curriculares que fortalezcan el proceso educativo, estableciendo una premisa, el diseño curricular, sin evaluación no es pedagógico. 


\section{REFERENCIAS BIBLIOGRÁFICAS}

1. Frida Días Barriga, (1987). Diseño curricular para la educación superior. Editorial Trillas.

2. Kenneth Delgado Santa Gadea, (2008). Evaluación en la educación superior. Unidad de Post Grado de la Facultad de Educación de la Universidad Nacional Mayor de San Marcos.

3. Santiago Castillo Arredondo, Jesús Cabrerizo Diago, (2010). Evaluación educativa de aprendizajes y competencias. Editorial Pearson.

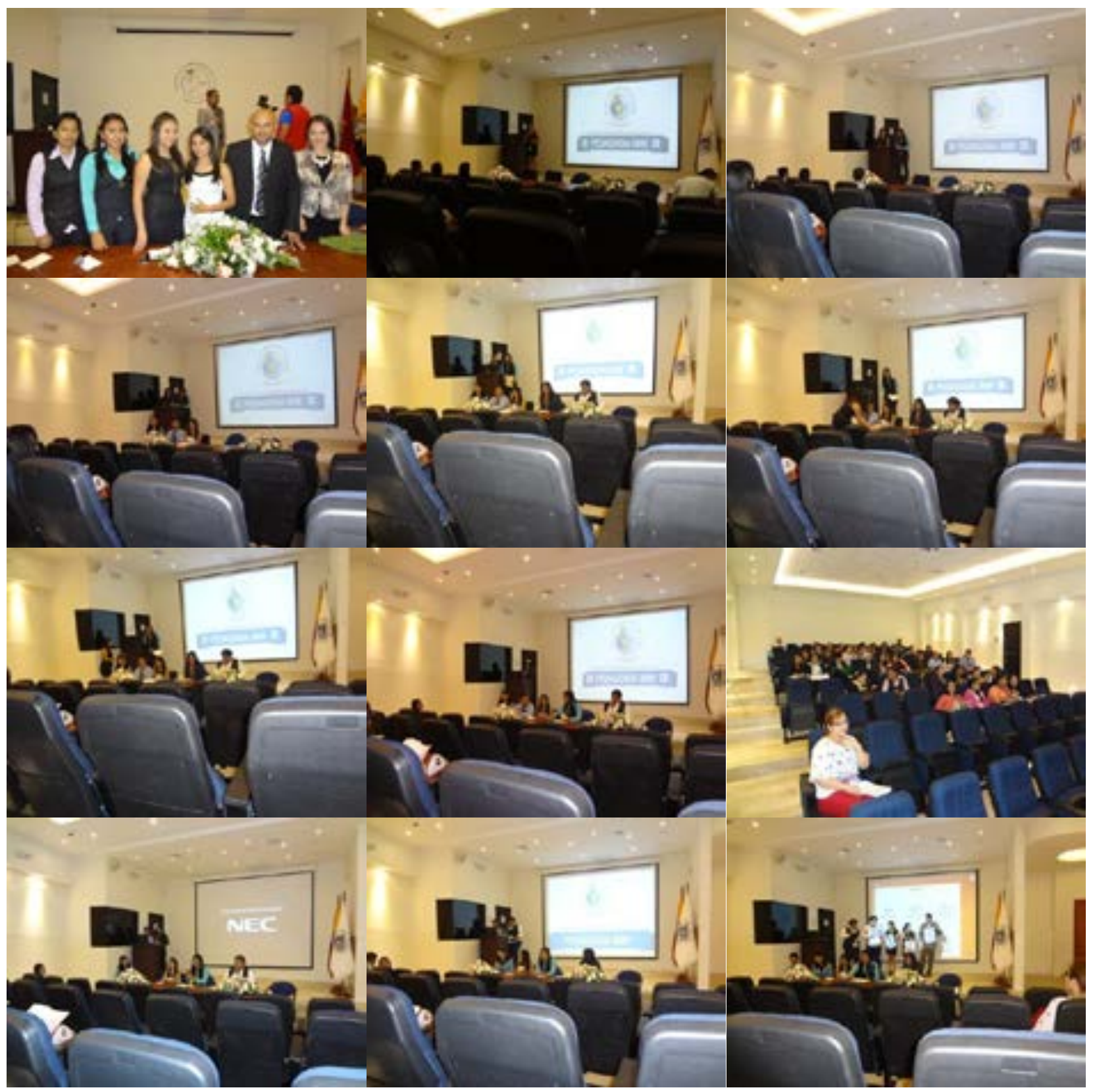

